\title{
ON THE (SUB)LOGARITHMIC PROPERTY OF THE POLE-, ZERO- AND ALGEBRAIC MULTIPLICITY OF OPERATOR FUNCTIONS
}

\author{
by G. PHILIP A. THIJSSE ${ }^{1}$ \\ (Received 12th December 1978)
}

This paper contains an extension of a result obtained by H. Bart, M. A. Kaashoek and D. C. Lay in (2). These authors studied the reduced algebraic multiplicity $R M\left(A ; \lambda_{0}\right)$ of a meromorphic operator function at a point $\lambda_{0} \in C$. They proved that under certain conditions this quantity has logarithmic behaviour, i.e.,

$$
R M\left(A B ; \lambda_{0}\right)=R M\left(A ; \lambda_{0}\right)+R M\left(B ; \lambda_{0}\right) .
$$

For more restricted cases such results had been proved by others, notably I. C. Gohberg and E. I. Sigal (see (4) and (5)). Here we shall prove that such a result also holds for a larger class of operator functions than the diagonable functions considered in (2). ${ }^{2}$

The reduced algebraic multiplicity is the difference of two quantities (not explicitly introduced in (2)), namely the reduced zero multiplicity $r m_{0}\left(A ; \lambda_{0}\right)$ and the pole multiplicity $m_{p}\left(A ; \lambda_{0}\right)$. We present a characterisation of these quantities in terms of spaces of germs of holomorphic vector functions. The pole multiplicity has sublogarithmic behaviour, i.e., the inequality $m_{p}\left(A B ; \lambda_{0}\right) \leqq m_{p}\left(A ; \lambda_{0}\right)+m_{p}\left(B ; \lambda_{0}\right)$ holds. We present necessary and sufficient conditions on $A$ and $B$ in order that $m_{p}\left(A B ; \lambda_{0}\right)=$ $m_{p}\left(A ; \lambda_{0}\right)+m_{p}\left(B ; \lambda_{0}\right)$ if all these quantities are finite. Such conditions are of interest in system theory (see (9)), and J. Vandewalle and P. Dewilde have obtained other conditions in order that $m_{p}$ has logarithmic behaviour; we explain the relationship between their conditions and ours. Finally, we prove that the reduced zero multiplicity has also sublogarithmic behaviour, albeit under rather restrictive conditions.

\section{Examples and characterisation of multiplicities}

In this paper we shall use without further explanation all notations from (2). Throughout, $X, Y$ and $Z$ will denote Banach spaces.

Let $A \in H\left(\lambda_{0}, L(X, Y)\right)$. We define the pole multiplicity $m_{p}\left(A ; \lambda_{0}\right)$, the zero multiplicity $m_{0}\left(A ; \lambda_{0}\right)$ and the reduced zero multiplicity $r m_{0}\left(A ; \lambda_{0}\right)$ of $A$ at $\lambda_{0}$ by

$$
m_{p}\left(A ; \lambda_{0}\right)=\sum_{m=-\infty}^{-1} \operatorname{dim} K_{m}\left[A ; \lambda_{0}\right]
$$

\footnotetext{
1 The research for this paper was done while the author was employed at the Vrije Universiteit in Amsterdam.

${ }^{2}$ This result was proved earlier in the author's thesis (8, Theorem IV, 3.7).
} 


$$
\begin{aligned}
m_{0}\left(A ; \lambda_{0}\right) & =\sum_{m=0}^{\infty} \operatorname{dim} H_{m}\left[A ; \lambda_{0}\right], \\
r m_{0}\left(A ; \lambda_{0}\right) & =\sum_{m=0}^{\infty} \operatorname{dim}\left(H_{m}\left[A ; \lambda_{0}\right] / H\left[A ; \lambda_{0}\right]\right) .
\end{aligned}
$$

These quantities were mentioned, but not considered explicitly, in (2). From formulae (2) and (3) in (2) one sees that the algebraic multiplicity $M\left(A ; \lambda_{0}\right)$ and the reduced algebraic multiplicity $R M\left(A ; \lambda_{0}\right)$ of $A$ at $\lambda_{0}$ are given by

$$
\begin{aligned}
M\left(A ; \lambda_{0}\right) & =m_{0}\left(A ; \lambda_{0}\right)-m_{p}\left(A ; \lambda_{0}\right), \\
R M\left(A ; \lambda_{0}\right) & =r m_{0}\left(A ; \lambda_{0}\right)-m_{p}\left(A ; \lambda_{0}\right) .
\end{aligned}
$$

First we present some examples concerning the zero multiplicity.

Example 1. Let $A(\lambda)=T_{0}+\lambda T_{1}+\ldots+\lambda^{n} T_{n}(\lambda \in C)$, where

$$
T_{0}, T_{1}, \ldots, T_{n} \in L(X, Y) \text {. }
$$

Then $m_{0}\left(A ; \lambda_{0}\right)$ is equal to the number of linearly independent solutions of the equation

$$
A\left(\frac{d}{d t}\right) x(t)=0
$$

which are of the form $x(t)=p(t) e^{\lambda_{0} t^{t}}$, where $p$ is a polynomial with coefficients in $X$.

A proof for this statement can be found in (8), Example II, 1.1. It relies on the observation that

$$
A\left(\frac{d}{d t}\right)\left(p_{0}+p_{1} t+\ldots+\frac{p_{m}}{m !} t^{m}\right) e^{\lambda_{0} t}=0
$$

implies that $p_{m} \in H_{m}\left[A ; \lambda_{0}\right]$ and that each element $p_{m} \in H_{m}\left[A ; \lambda_{0}\right]$ gives rise to a chain $p_{m}, p_{m-1}, \ldots, p_{0}$ in $X$ such that (1) holds. This relationship between the zero multiplicity and the number of solutions of a differential equation was already indicated by $M$. V. Keldysh (7).

Example 2. Let $A(\lambda)=T-\lambda I$, where $T \in L(X)$ and $I$ denotes the identity on $X$. Then for $m \geqq 0$ one has

$$
H_{m}\left[A ; \lambda_{0}\right]=N\left(A\left(\lambda_{0}\right)\right) \cap R\left(A\left(\lambda_{0}\right)^{m}\right) \cong N\left(A\left(\lambda_{0}\right)^{m+1}\right) / N\left(A\left(\lambda_{0}\right)^{m}\right)
$$

and hence

$$
m_{0}\left(A ; \lambda_{0}\right)=\operatorname{dim}\left(\bigcup_{m=0}^{\infty} N\left(A\left(\lambda_{0}\right)^{m}\right)\right)
$$

The pole multiplicity plays a role in system theory where it is called the polar degree (see, e.g., (10)).

For an arbitrary Banach space $Z$ we denote through $H(Z)$ the space of germs of $Z$-valued functions which are holomorphic at the (fixed) complex point $\lambda_{0}$. With $M(Z)$ we denote the space of germs of $Z$-valued functions which are meromorphic at $\lambda_{0}$. If $A \in M(L(X, Y))$, then $A$ induces a linear map $\hat{A}: M(X) \rightarrow M(Y)$ defined by $(\hat{A} \phi)(\lambda)=$ 
$A(\lambda) \phi(\lambda)$ for $\lambda$ near $\lambda_{0}$. Our first theorem relates $m_{p}\left(A ; \lambda_{0}\right)$ and $r m_{0}\left(A ; \lambda_{0}\right)$ to properties of $\hat{A}$.

Theorem 1. Let $A \in H\left(\lambda_{0}, L(X, Y)\right)$ be meromorphic at $\lambda_{0}$. Then

$$
\begin{aligned}
m_{p}\left(A ; \lambda_{0}\right) & =\operatorname{dim} \frac{H(X)}{\left(\hat{A}^{-1}[H(Y)] \cap H(X)\right)} \\
& =\operatorname{dim} \frac{\hat{A}[H(X)]}{(\hat{A}[H(X)] \cap H(Y))} .
\end{aligned}
$$

If, moreover, $A$ is finite-meromorphic at $\lambda_{0}, R\left(A_{0}\right)$ is closed and $r m_{0}\left(A ; \lambda_{0}\right)<\infty$ then

$$
r m_{0}\left(A ; \lambda_{0}\right)=\operatorname{dim} \frac{(H(Y) \cap \hat{A}[M(X)])}{(H(Y) \cap \hat{A}[H(X)])} .
$$

If, in addition to the conditions mentioned above, we have $H\left[A ; \lambda_{0}\right]=\{0\}$, then

$$
r m_{0}\left(A ; \lambda_{0}\right)=m_{0}\left(A ; \lambda_{0}\right)=\operatorname{dim} \frac{\hat{A}^{-1}[H(Y)]}{\left(\hat{A}^{-1}[H(Y)] \cap H(X)\right)} .
$$

Proof. The second identity in (2) follows from the observation that $\phi_{1}, \ldots, \phi_{n} \in$ $H(X)$ are linearly independent modulo $\hat{A}^{-1}[H(Y)] \cap H(X)$ if and only if $\hat{A} \phi_{1}, \ldots, \hat{A} \phi_{n}$ are linearly independent modulo $\hat{A}[H(X)] \cap H(Y)$. To prove the first identity in (2), assume that $m_{p}\left(A ; \lambda_{0}\right)$ is finite but non-zero. Let $l=-\nu\left(A ; \lambda_{0}\right)$. Then $l>0$. Choose a basis $y_{1}, \ldots, y_{k}$ for $K_{-1}\left[A ; \lambda_{0}\right]$ such that there exist integers $k=i_{1} \geqq i_{2} \geqq \ldots \geqq i_{l}>0$ with

$$
K_{-m}\left[A ; \lambda_{0}\right]=\operatorname{sp}\left\{y_{1}, \ldots, y_{i_{m}}\right\}
$$

for $m=1,2, \ldots, l$. Set $i_{l+1}=0$ and assume that $i_{s} \geqq j>i_{s+1}$. Then there exists $\phi_{j} \in H(X)$ such that $\nu\left(\phi_{j} ; \lambda_{0}\right) \geqq s$ and $A \phi_{j} \rightarrow y_{j}$. Define $\phi_{j i}(i=1,2, \ldots, s)$ by

$$
\phi_{j i}(\lambda)=\left(\lambda-\lambda_{0}\right)^{-i} \phi_{j}
$$

Then $\phi_{j i} \in H(X)$ for $j=i_{s+1}+1, \ldots, i_{s}, i=1,2, \ldots, l$. It can be proved (see (8), Example IV, 1.3) that

$$
\left\{\hat{A} \phi_{j i} \mid j=i_{s+1}+1, \ldots, i_{s}, i=1,2, \ldots, s, s=1,2, \ldots, l\right\}
$$

forms a basis for $\hat{A}[H(X)]$ modulo $\hat{A}[H(X)] \cap H(Y)$. Note the the number of elements in (5) is given by

$$
\sum_{s=1}^{l} s .\left(i_{s}-i_{s+1}\right)=\sum_{s=1}^{l} \operatorname{dim} K_{-s}\left[A ; \lambda_{0}\right]=m_{p}\left(A ; \lambda_{0}\right)
$$

This proves our claim.

Next we prove equality (3). We restrict ourselves to the case where $0<r m_{0}\left(A ; \lambda_{0}\right)$. Let $q=\min \left\{n \mid H_{n}\left[A ; \lambda_{0}\right]=H\left[A ; \lambda_{0}\right]\right\}$. As

$$
\operatorname{dim}\left(H_{n}\left[A ; \lambda_{0}\right] / H\left[A ; \lambda_{0}\right]\right)=\operatorname{dim}\left(K\left[A ; \lambda_{0}\right] / K_{n}\left[A ; \lambda_{0}\right]\right)
$$

(cf. (1), formula (1-2)) we have that $K\left[A ; \lambda_{0}\right]=K_{q}\left[A ; \lambda_{0}\right]$; moreover,

$$
r m_{0}\left(A ; \lambda_{0}\right)=\sum_{i=0}^{q-1} \operatorname{dim} \frac{K_{q}\left[A ; \lambda_{0}\right]}{K_{i}\left[A ; \lambda_{0}\right]}
$$


There exist vectors $y_{1}, \ldots, y_{k} \in K_{q}\left[A ; \lambda_{0}\right]$, which are linearly independent modulo $K_{0}\left[A ; \lambda_{0}\right]$, and integers $0 \leqq i_{1} \leqq \ldots \leqq i_{q}=k$ such that

$$
K_{j}\left[A ; \lambda_{0}\right]=K_{0}\left[A ; \lambda_{0}\right] \oplus \operatorname{sp}\left\{y_{1}, \ldots, y_{i_{i}}\right\}
$$

for $j=1,2, \ldots, q$. Set $i_{0}=0$. For each $y_{j}\left(i_{s-1}<j \leqq i_{s}\right)$ there exists $\phi_{j} \in M(X)$ such that $\hat{A} \phi_{j} \rightarrow y_{j}$ and $\nu\left(\phi_{j} ; \lambda_{0}\right)=-s$. Note that the set

$$
\left\{\hat{A}\left(\lambda-\lambda_{0}\right)^{s} \phi_{j} \mid j=i_{s}, \ldots, k, s=0,1, \ldots, q-1\right\}
$$

has exactly $r m_{0}\left(A ; \lambda_{0}\right)$ elements. It is not difficult to prove (cf. (8), Example IV, 1.4) that (6) is a subset of $H(Y) \cap \hat{A}[M(X)]$ which is linearly independent modulo $H(Y) \cap \hat{A}[H(X)]$. This shows that

$$
r m_{0}\left(A ; \lambda_{0}\right) \leqq \operatorname{dim} \frac{(H(Y) \cap \hat{A}[M(X)])}{(H(Y) \cap \hat{A}[H(X)])}
$$

In order to prove the reverse inequality we need our extra assumptions on $A$. These enable us to write $A=S B$, where $S$ is holomorphic at $\lambda_{0}$ and $K_{0}\left[S ; \lambda_{0}\right]=K\left[S ; \lambda_{0}\right]=K\left[A ; \lambda_{0}\right]$, whereas $B$ is of the form

$$
B(\lambda)=\left(I-P_{1}+\left(\lambda-\lambda_{0}\right)^{s_{1}} P_{1}\right) \ldots\left(I-P_{n}+\left(\lambda-\lambda_{0}\right)^{s_{n}} P_{n}\right)
$$

where all $P_{i}$ are degenerate projections of $X$ and all $s_{i}$ are integers (cf: (8)). According to Theorem II, 3.1 in (8) we may choose $B$ such that $m_{0}\left(B ; \lambda_{0}\right)=r m_{0}\left(A ; \lambda_{0}\right)$. As $B(\lambda)$ is bijective for $\lambda \neq \lambda_{0}$, the operator $\hat{B}$ maps $M(X)$ onto itself in a bijective way. From Proposition I, 3.7 in (8) we have

$$
H(Y) \cap \hat{A}[M(X)]=H(Y) \cap \hat{S}[M(X)]=H(Y) \cap \hat{S}[H(X)] .
$$

Hence, with $\hat{A}=\hat{S} \hat{B}$,

$$
\begin{aligned}
& \operatorname{dim} \frac{(H(Y) \cap \hat{A}[M(X)])}{(H(Y) \cap \hat{A}[H(X)])} \\
= & \operatorname{dim} \frac{(H(Y) \cap \hat{S}[H(X)])}{(H(Y) \cap \hat{A}[H(X)])} \\
\leqq & \operatorname{dim} \frac{(H(X) \cap \hat{B}[M(X)])}{(H(X) \cap \hat{B}[H(X)])} \\
= & \operatorname{dim} \frac{\hat{B}^{-1}[H(X)]}{\left(H(X) \cap \hat{B}^{-1}[H(X)]\right)}=m_{p}\left(B^{-1} ; \lambda_{0}\right)
\end{aligned}
$$

According to Lemma II, 1.4 in $(8)$ we have $m_{p}\left(B^{-1} ; \lambda_{0}\right)=m_{0}\left(B ; \lambda_{0}\right)$. This completes the proof of (3).

Finally, if $H\left[A ; \lambda_{0}\right]=\{0\}$, then $m_{0}\left(A ; \lambda_{0}\right)=r m_{0}\left(A ; \lambda_{0}\right)$, and the other assumptions on $A$ assure that $\hat{A}$ is injective, as $A(\lambda)$ is injective for all $\lambda$ in some deleted neighbourhood of $\lambda_{0}$ (cf. (1), Corollary 6.4). So (4) follows immediately from (3).

The characterisations obtained in Theorem 1 will be used for the proof of the (sub)logarithmic behaviour of the multiplicities. 


\section{2. (Sub)logarithmic behaviour of the pole multiplicity}

In this section we present a new proof for the sublogarithmic property of the pole multiplicity and we give necessary and. sufficient conditions in order that $m_{p}\left(A B ; \lambda_{0}\right)$ behaves logarithmically. We use these conditions in order to derive a result on the stability of this logarithmic behaviour under holomorphic perturbations on $A$ and $B$.

Theorem 2. Let $A \in H\left(\lambda_{0}, L(Y, Z)\right.$ and $B \in H\left(\lambda_{0}, L(X, Y)\right)$ be finite-meromorphic at $\lambda_{0}$. Then

$$
m_{p}\left(A B ; \lambda_{0}\right) \leqq m_{p}\left(A ; \lambda_{0}\right)+m_{p}\left(B ; \lambda_{0}\right) .
$$

Further, $m_{p}\left(A B ; \lambda_{0}\right)=m_{p}\left(A ; \lambda_{0}\right)+m_{p}\left(B ; \lambda_{0}\right)$ if and only if the following conditions are satisfied

(i) $H_{0}\left[A ; \lambda_{0}\right] \cap K_{-1}\left[B ; \lambda_{0}\right]=\{0\}$

(ii) $H_{-1}\left[A ; \lambda_{0}\right]+K_{0}\left[B ; \lambda_{0}\right]=Y$.

Proof. Set $H_{0}=H(X), \quad H_{1}=H(X) \cap \hat{B}^{-1}[H(Y)], \quad H_{2}=H(X) \cap\left(\hat{A} \hat{B}^{-1}[H(Z)]\right.$, $H_{3}=H_{1} \cap H_{2}$. As $\operatorname{Ker}\left(\left.\hat{B}\right|_{H(X)}\right) \subset H_{3}$ and $\hat{B}\left[H_{3}\right]=\hat{B}\left[H_{1}\right] \cap \hat{A}^{-1}[H(Z)] \cap H(Y)$, we have

$$
\begin{aligned}
& \operatorname{dim} \frac{H_{1}}{H_{3}}=\operatorname{dim} \frac{\hat{B}\left[H_{1}\right]}{\hat{B}\left[H_{3}\right]} \\
= & \operatorname{dim} \frac{\hat{B}\left[H_{1}\right]}{\left(\hat{B}\left[H_{1}\right] \cap \hat{A}^{-1}[H(Z)] \cap H(Y)\right)} \\
\leqq & \operatorname{dim} \frac{H(Y)}{\left(H(Y) \cap \hat{A}^{-1}[H(Z)]\right)}=m_{p}\left(A ; \lambda_{0}\right) .
\end{aligned}
$$

Thus

$$
\begin{aligned}
& m_{p}\left(A B ; \lambda_{0}\right)=\operatorname{dim} \frac{H_{0}}{H_{2}} \leqq \operatorname{dim} \frac{H_{0}}{H_{3}} \\
= & \operatorname{dim} \frac{H_{0}}{H_{1}}+\operatorname{dim} \frac{H_{1}}{H_{3}} \leqq \operatorname{dim} \frac{H_{0}}{H_{1}}+m_{p}\left(A ; \lambda_{0}\right) \\
= & m_{p}\left(B ; \lambda_{0}\right)+m_{p}\left(A ; \lambda_{0}\right),
\end{aligned}
$$

which proves (7). At the same time we see that equality holds in (7) if and only if

(i) $\mathrm{H}_{2}=\mathrm{H}_{3}$

(ii) $\frac{\hat{B}\left[H_{1}\right]}{\left(\hat{B}\left[H_{1}\right] \cap \hat{A}^{-1}[H(Z)] \cap H(Y)\right)} \cong \frac{H(Y)}{\left(H(Y) \cap \hat{A}^{-1}[H(Z)]\right)}$

It is not difficult to see that conditions (9) are satisfied and only if

(i) $(\hat{B}[H(X)]+H(Y)) \cap\left(\hat{A}^{-1}[H(Z)]+H(Y)\right)=H(Y)$

(ii) $(\hat{B}[H(X)] \cap H(Y))+\left(\hat{A}^{-1}[H(Z)] \cap H(Y)\right)=H(Y)$

Indeed, $\left(9\right.$, ii) is satisfied if and only if $\hat{B}\left[H_{1}\right]+\left(\hat{A}^{-1}[H(Z)] \cap H(Y)\right)=H(Y)$. As $\hat{B}\left[H_{1}\right]=$ $\hat{B}[H(X)] \cap H(Y)$, this proves that $(9$, ii) and $(10$, ii) are equivalent; further, if $(9, \mathrm{i})$ is not 
satisfied, then $\phi \in H_{2} \backslash H_{1}$ exists, i.e., there is a $\phi \in H(X)$ such that $\hat{B} \phi \notin H(Y)$ and $\hat{A} \hat{B} \phi \in H(Z)$. Then $\hat{B} \phi \in\left(\hat{B}[H(X)] \cap \hat{A}^{-1}[H(Z)]\right) \backslash H(Y)$, i.e., $(10$, i) does not hold. Finally (9) implies $(10, \mathrm{i})$. To see this let $\psi \in(\hat{B}[H(X)]+H(Y)) \cap\left(\hat{A}^{-1}[H(Z)]+H(Y)\right)$ and assume that $\psi \notin H(Y)$. Without loss of generality we may take $\psi=\hat{B} \phi$ with $\phi \in H(X)$. Then $\phi \notin H_{1}$. From $(9$, ii) (which implies $(10$, ii)) it follows that there exist $\theta \in H(X), \eta \in \hat{A}^{-1}[H(Z)] \cap H(Y)$ such that $\hat{B} \theta \in \hat{B}[H(X)] \cap H(Y)$ and

$$
\hat{B} \phi-\hat{B} \theta-\eta \in \hat{A}^{-1}[H(Z)] ;
$$

in particular, $\hat{B}(\phi-\theta) \in \hat{A}^{-1}[H(Z)]$ and $\phi-\theta \in H_{2} \backslash H_{1}$, thus contradicting $(9, \mathrm{i})$.

We complete the proof by showing that $(10, \mathrm{i})$ is equivalent with $(8, \mathrm{i})$ and $(10, \mathrm{ii})$ with $(8$, ii). First, assume that $(10$, i) does not hold, i.e., there exists $\psi \in$ $\left((\hat{B}[H(X)]+H(Y)) \cap\left(\hat{A}^{-1}[H(Z)]+H(Y)\right)\right) \backslash H(Y)$. Without loss of generality, $\psi \epsilon$ $\hat{A}^{-1}[H(Z)]$ and $\psi=\hat{B} \theta+\eta$, with $\theta \in H(X)$ and $\eta \in H(Y)$. Let $-k=\nu\left(\psi ; \lambda_{0}\right)$. Then $\left(\lambda-\lambda_{0}\right)^{k} \psi(\lambda) \rightarrow y \neq 0$, i.e., $B(\lambda)\left(\lambda-\lambda_{0}\right)^{k} \theta(\lambda) \rightarrow y$. As $k>0$ this proves $y \in K_{-1}\left[B ; \lambda_{0}\right]$. Moreover, $\nu\left(A(\lambda)\left(\lambda-\lambda_{0}\right)^{k} \psi(\lambda) ; \lambda_{0}\right)=k+\nu\left(\hat{A} \psi ; \lambda_{0}\right) \geqq k$, so $y \in H_{0}\left[A ; \lambda_{0}\right]$. This proves that (8.1) does not hold. If, conversely, $0 \neq y \in H_{0}\left[A ; \lambda_{0}\right] \cap K_{-1}\left[B ; \lambda_{0}\right]$, then there exist $\psi \in H(Y), \theta \in H(X)$ such that $\psi(\lambda) \rightarrow y, \nu\left(\theta ; \lambda_{0}\right) \geqq 1,(\hat{B} \theta)(\lambda) \rightarrow y$ and $\nu\left(\hat{A} \psi ; \lambda_{0}\right) \geqq 1$. Then $\left(\lambda-\lambda_{0}\right)^{-1} \psi \in \hat{A}^{-1}[H(Z)],\left(\lambda-\lambda_{0}\right)^{-1} \hat{B} \theta \in \hat{B}[H(X)]$, so

$$
\left(\lambda-\lambda_{0}\right)^{-1} y \in\left((\hat{B}[H(X)]+H(Y)) \cap\left(\hat{A}^{-1}[H(Z)]+H(Y)\right)\right) \backslash H(Y)
$$

and $(10, i)$ does not hold.

Next, assume that $(10$, ii) holds. Then the function $y(\lambda) \equiv y$ can be represented as $y=\hat{B} \theta+\eta$, where $\theta \in H(X), \eta \in H(Y)$ and $\hat{A} \eta \in H(Z)$. Then $\eta \rightarrow \eta_{0} \in H_{-1}\left[A ; \lambda_{0}\right]$, $\hat{B} \theta \rightarrow y-\eta_{0} \in K_{0}\left[B ; \lambda_{0}\right]$, which proves $(8$, ii). Finally, assume that $(8$, ii) holds. Let $y \in H(Y)$ and $y(\lambda) \rightarrow y_{0}$. Then $y_{0}=u_{0},+\eta_{0}$, where $\hat{B} \theta_{0} \rightarrow u_{0}, \psi_{0} \rightarrow \eta_{0}$ for some $\theta_{0} \in$ $H(X), \quad \psi_{0} \in \hat{A}^{-1}[H(Z)] \cap H(Y)$. Consider $\left(\lambda-\lambda_{0}\right)^{-1}\left(y(\lambda)-\left(\hat{B} \theta_{0}\right)(\lambda)-\psi_{0}(\lambda)\right) \in H(Y)$. Repeating the above construction we obtain $\theta_{1} \in H(X), \psi_{1} \in H(Y) \cap \hat{A}^{-1}[H(Z)]$ such that

$$
\nu\left(y-\hat{B} \theta_{0}-\psi_{0}-\left(\lambda-\lambda_{0}\right) \hat{B} \theta_{1}-\left(\lambda-\lambda_{0}\right) \psi_{1} ; \lambda_{0}\right) \geqq 2 .
$$

Let $q=-\nu\left(A ; \lambda_{0}>0\right.$. Repeating the described procedure we obtain $\theta_{0}, \theta_{1}, \ldots, \theta_{q-1} \in$ $H(X), \psi_{0}, \psi_{1}, \ldots, \psi_{q-1} \in H(Y) \cap \hat{A}^{-1}[H(Z)]$ such that $\hat{B} \theta_{0}, \hat{B} \theta_{1}, \ldots, \hat{B} \theta_{q-1} \in H(Y)$ and

Then

$$
\nu\left(y-\hat{B}\left(\sum_{j=0}^{q-1}\left(\lambda-\lambda_{0}\right)^{j} \theta_{j}\right)-\sum_{j=0}^{q-1}\left(\lambda-\lambda_{0}\right)^{j} \psi_{j} ; \lambda_{0}\right) \geqq q .
$$

$$
u=y-\hat{B}\left(\sum_{j=0}^{a-1}\left(\lambda-\lambda_{0}\right)^{j} \theta_{j}\right)-\sum_{j=0}^{q-1}\left(\lambda-\lambda_{0}\right)^{j} \psi_{j} \in \hat{A}^{-1}[H(Z)]
$$

and so $y \in(\hat{B}[H(X)] \cap H(Y))+\left(\hat{A}^{-1}[H(Z)] \cap H(Y)\right)$. This completes the proof.

Let $A, C \in H\left(\lambda_{0}, L(Y, Z)\right)$ and $B, D \in H\left(\lambda_{0}, L(X, Y)\right)$ be finite-meromorphic at $\lambda_{0}$, and assume that conditions (8) are satisfied for $A$ and $B$, i.e., $m_{p}\left(A B ; \lambda_{0}\right)$ has logarithmic behaviour. It is interesting to ask whether $m_{p}\left((A+C)(B+D) ; \lambda_{0}\right)$ has logarithmic behaviour too. Conditions (8) reduce this question to a relatively simple question on $H_{i}\left[A+C ; \lambda_{0}\right]$ and $K_{i}\left[B+D ; \lambda_{0}\right]$ for $i=-1,0$. For example, if $C$ and $D$ are holomorphic at $\lambda_{0}$ and have a zero at $\lambda_{0}$, then $H_{i}\left[A+C ; \lambda_{0}\right]=H_{i}\left[A ; \lambda_{0}\right], K_{i}\left[B+D ; \lambda_{0}\right]=K_{i}\left[B ; \lambda_{0}\right]$ for 
$i=-1,0$ and $m_{p}\left((A+C)(B+D) ; \lambda_{0}\right)$ has logarithmic behaviour. Theorem 3 extends this observation to the case where $C$ and $D$ are holomorphic at $\lambda_{0}$ and their values in $\lambda_{0}$ are small in norm.

Theorem 3. Let $A, B, C$ and $D$ be as above and assume that $m_{p}\left(A B ; \lambda_{0}\right)=$ $m_{p}\left(A ; \lambda_{0}\right)+m_{p}\left(B ; \lambda_{0}\right)$. If the constant terms of the Laurent expansions of $A$ and $B$ are operators with closed range, then there exists $\varepsilon>0$ such that if $C, D$ are holomorphic at $\lambda_{0}$ and $\max \left\{\left\|C\left(\lambda_{0}\right)\right\|,\left\|D\left(\lambda_{0}\right)\right\|\right\}<\varepsilon$, then

$$
\left.m_{p}\left((A+C)(B+D) ; \lambda_{0}\right)=m_{p}\left(A+C ; \lambda_{0}\right)+m_{p} B+D ; \lambda_{0}\right) .
$$

Proof. As $C, D$ are holomorphic at $\lambda_{0}$ we have $H_{-1}\left[A+C ; \lambda_{0}\right]=H_{-1}\left[A ; \lambda_{0}\right]$, $K_{-1}\left[B+D ; \lambda_{0}\right]=K^{-1}\left[B ; \lambda_{0}\right]$. Moreover, $K_{0}\left[B ; \lambda_{0}\right]$ is closed, as the constant term of the Laurent expansion of $B$ at $\lambda_{0}$ has closed range. Let $\varepsilon_{1}$ be given by

$$
\varepsilon_{1}=\min \left\{\gamma\left(K_{0}\left[B ; \lambda_{0}\right], H_{-1}\left[A ; \lambda_{0}\right]\right), \gamma\left(K_{-1}\left[B ; \lambda_{0}\right], H_{0}\left[A ; \lambda_{0}\right]\right)\right\},
$$

where $\gamma$ denotes the (asymmetric) minimal gap (see (6), Ch. IV, §4.1, formula 4.4). One can prove that if $\varepsilon$ is chosen sufficiently small, then

$$
\max \left\{\delta\left(K_{0}\left[B ; \lambda_{0}\right], K_{0}\left[B+D ; \lambda_{0}\right]\right), \delta\left(H_{0}\left[A+C ; \lambda_{0}\right], H_{0}\left[A ; \lambda_{0}\right]\right)\right\}<\varepsilon_{1},
$$

where $\delta$ is the asymmetric distance (see (6), Ch. IV, $\$ 2.1$, Formula (2.1)). It follows from Theorem IV, 4.24 in (6) that

$$
H_{-1}\left[A ; \lambda_{0}\right]+K_{0}\left[B+D ; \lambda_{0}\right]=Y, \quad H_{0}\left[A+C ; \lambda_{0}\right] \cap K_{-1}\left[B ; \lambda_{0}\right]=\{0\}
$$

if (11) is satisfied. This completes the proof.

In (9) J. Vandewalle and P. Dewilde present necessary and sufficient conditions for the logarithmic behaviour of the pole multiplicity, or polar degree as they call it $(\mathbf{9}$, conditions (73)). These conditions are entirely different from our conditions $(\mathbf{8})$, but we shall explain that our conditions $(10)$ are in fact a variant of their conditions (73). To this end, let $P(X)$ denote the space of germs of principal parts at $\lambda_{0}$. Then $M(X)=H(X) \oplus P(X)$. If $F(X)$ denotes the projection of $M(X)$ onto $H(X)$ along $P(X)$ and $E(X)=I-F(X)$ then conditions (10) may also be expressed as

(i) $(\operatorname{Im}(\hat{B} F(X))+\operatorname{Im} F(Y)) \cap(\operatorname{Ker}(E(Z) \hat{A})+\operatorname{Im} F(Y))=\operatorname{Im} F(Y)$,

(ii) $(\operatorname{Im}(\hat{B} F(X)) \cap \operatorname{Im} F(Y))+(\operatorname{Ker}(E(Z) \hat{A}) \cap \operatorname{Im} F(Y))=\operatorname{Im} F(Y)$.

In this formulation our conditions are already much like conditions (73) in (9). There, however, a notion of orthogonality is involved. In our setting this can be achieved by defining

$$
\langle\phi, x\rangle=\frac{1}{2 \pi i} \int_{\Gamma} \frac{\langle\phi(\lambda), x(\lambda)\rangle}{\lambda-\lambda_{0}} d \lambda
$$

for $\phi \in M\left(X^{*}\right), x \in M(X)$ and $\Gamma$ a sufficiently small circle around $\lambda_{0}$. We define $(\hat{A})^{*}=\hat{A}^{*}$ and with $E(X)^{*}$ we denote the projection of $M\left(X^{*}\right)$ onto the subspace of all germs of holomorphic $X^{*}$-valued functions which have a zero in $\lambda_{0}$ along the subspace of 
all polynomials in $\left(\lambda-\lambda_{0}\right)^{-1}$. Further, $F(X)^{*}=I-E(X)^{*}$. Note that $E(X)^{*} \neq E\left(X^{*}\right)$. It is not difficult to prove that $\hat{A}^{*}, E(X)^{*}, F(X)^{*}$ behave like conjugates. i.e.,

$$
\left\langle C^{*} D^{*} \phi, x\right\rangle=\langle\phi, D C x\rangle,
$$

where $C, D$ are operators like $\hat{A}, E(X), F(X)$. We may also define annihilators setting

$$
M^{\perp}=\left\{\phi \in M\left(X^{*}\right) \mid\langle\phi, m\rangle=0 \text { for all } m \in M\right\}
$$

if $M \subset M(X)$ and

$$
{ }_{\perp} N=\{x \in M(X) \mid\langle n, x\rangle=0 \text { for all } n \in N\}
$$

if $N \subset M\left(X^{*}\right)$. From these annihilators the usual relations like $(M+N)^{\perp}=M^{\perp} \cap N^{\perp}$, $(M \cap N)^{\perp} \supset M^{\perp}+N^{\perp}$ are valid. Moreover

$$
\operatorname{Ker} C={ }^{\perp} \operatorname{Im} C^{*},
$$

where $C$ is an operator like $\hat{A}, E(X), F(X)$ or a product of these. Using these notions, conditions (12) can be rewritten as

(i) ${ }^{\perp}\left(\operatorname{Im}\left(\hat{A}^{*} E(Z)^{*}\right) \cap \operatorname{Im}\left(E(Y)^{*}\right)\right) \cap(\operatorname{Im}(\hat{B} F(X))+\operatorname{Im} F(Y))=\operatorname{Im} F(Y)$

(ii) $\left(\operatorname{Im}\left(\hat{A}^{*} E(Z)^{*}\right)+\operatorname{Im}\left(E(Y)^{*}\right)\right) \cap(\operatorname{Im}(\hat{B} F(X)) \cap \operatorname{Im} F(Y))^{\perp}=\operatorname{Im}\left(E(Y)^{*}\right)$

and if $A$ and $B$ are both finite-meromorphic then conditions (13) are in fact equivalent with conditions (12), and therefore also with conditions (8). Conditions (13) are, however, identical to conditions (73) in (9).

\section{Logarithmic behaviour of the reduced algebraic multiplicity and sublogarithmic behaviour of the reduced zero multiplicity}

In this section we prove an extension of Theorem 2 in (2). First we deal with the special case where $A(\lambda)$ is an injective $\Phi^{-}$-operator and $B(\lambda)$ a surjective $\Phi^{+}$-operator for $\lambda$ in a deleted neighbourhood of $\lambda_{0}$ (cf. (8), Proposition IV, 3.5), as the general case can be reduced to this case.

Proposition 1. Let $A \in H\left(\lambda_{0}, L(Y, Z)\right)$ and $B \in H\left(\lambda_{0}, L(X, Y)\right)$ be finitemeromorphic at $\lambda_{0}$ and assume that the constant terms of the Laurent expansions of $A$ and $B$ at $\lambda_{0}$ are semi-Fredholm operators. If $A(\lambda)$ is an injective $\Phi^{-}$-operator and $B(\lambda)$ a surjective $\Phi^{+}$-operator for $\lambda$ in a deleted neighbourhood of $\lambda_{0}$ then

$$
R M\left(A B ; \lambda_{0}\right)=M\left(A ; \lambda_{0}\right)+R M\left(B ; \lambda_{0}\right) .
$$

Proof. Observe that both $A$ and $B$ have finite zero multiplicity as for both operator functions the constant term of the Laurent expansion at $\lambda_{0}$ is a semi-Fredholm operator. It is not difficult to prove that $A B$ has finite reduced zero multiplicity as well (cf. (8), Lemma IV, 3.4), so the characterisations of Theorem 1 hold for $A, B$ and $A B$. Note that $\hat{A}$ is injective, so (4) holds for $m_{0}\left(A ; \lambda_{0}\right)$; it is well-known that $\hat{B}$ is surjective (cf. (3), Lemma 1.7 and (8), Theorem II, 3.1), so $\hat{B}[M(X)]=M(Y)$. Formula (14) can be verified by direct 
calculation. For simplicity we use the following notation: $H=H(Y), K=\hat{A}^{-1}[H(Z)]$, $L=\hat{B}[H(X)]$. Then

$$
\begin{aligned}
M\left(A ; \lambda_{0}\right) & =\operatorname{dim} \frac{K}{(H \cap K)}-\operatorname{dim} \frac{H}{(H \cap K)}, \\
R M\left(B ; \lambda_{0}\right) & =\operatorname{dim} \frac{H}{(H \cap L)}-\operatorname{dim} \frac{L}{(H \cap L)}, \\
R M\left(A B ; \lambda_{0}\right) & =\operatorname{dim} \frac{(H(Z) \cap \hat{A} \hat{B}[M(X)])}{(H(Z) \cap \hat{A} \hat{B}[H(X)])}-\operatorname{dim} \frac{\hat{A} \hat{B}[H(X)]}{(H(Z) \cap \hat{A} \hat{B}[H(X)])} .
\end{aligned}
$$

As $\hat{A}$ is injective and $\hat{B}$ surjective this reduces to

$$
R M\left(A B ; \lambda_{0}\right)=\operatorname{dim} \frac{K}{(K \cap L)}-\operatorname{dim} \frac{L}{(K \cap L)} .
$$

Note that (15), (16) and (17) remain valid if all denominators are replaced by $H \cap K \cap L$. Thus we obtain

$$
\begin{aligned}
M\left(A ; \lambda_{0}\right)+R M\left(B ; \lambda_{0}\right)= & \operatorname{dim} \frac{K}{(H \cap K \cap L)}-\operatorname{dim} \frac{H}{(H \cap K \cap L)} \\
& +\operatorname{dim} \frac{H}{(H \cap K \cap L)}-\operatorname{dim} \frac{L}{(H \cap K \cap L)} \\
= & \operatorname{dim} \frac{K}{(H \cap K \cap L)}-\operatorname{dim} \frac{L}{(H \cap K \cap L)}=R M\left(A B ; \lambda_{0}\right)
\end{aligned}
$$

and the proof is complete.

Theorem 4. Let $A \in H\left(\lambda_{0}, L(Y, Z)\right)$ and $B \in H\left(\lambda_{0}, L(X, Y)\right)$ be finitemeromorphic at $\lambda_{0}$, and assume that the constant terms of the Laurent expansions of $A$ and $B$ at $\lambda_{0}$ are operators with closed range and that both $r m_{0}\left(A ; \lambda_{0}\right)$ and $r m_{0}\left(B ; \lambda_{0}\right)$ are finite. If $K\left[B ; \lambda_{0}\right] \oplus H\left[A ; \lambda_{0}\right]=Y$ then $A B$ is finite-meromorphic at $\lambda_{0}$, the constant term of its Laurent expansion at $\lambda_{0}$ has closed range, its reduced zero multiplicity is finite and

$$
R M\left(A B ; \lambda_{0}\right)=R M\left(A ; \lambda_{0}\right)+R M\left(B ; \lambda_{0}\right) .
$$

Proof. All statements, except formula (18) follow from Lemma IV, 3.4 in (8). As $K\left[B ; \lambda_{0}\right]$ is a complemented subspace of $Y$ there exists an operator function $E$, holomorphic at $\lambda_{0}$ and with invertible values in $L(Y)$ such that $E(\lambda) K[B ; \lambda]=K\left[B ; \lambda_{0}\right]$. Put $M=K\left[B ; \lambda_{0}\right]$. For $\lambda$ in a neighbourhood of $\lambda_{0}$ we may consider $E(\lambda) B(\lambda)$ as an element of $H\left(\lambda_{0}, L(X, M)\right)$. Define $A_{1}$ by $A_{1}(\lambda) E(\lambda)^{-1} \mid M$. Then $A_{1}$ and $E B$ satisfy the conditions of Proposition 1, so

$$
R M\left(A B ; \lambda_{0}\right)=R M\left(A_{1} E B ; \lambda_{0}\right)=M\left(A_{1} ; \lambda_{0}\right)+R M\left(E B ; \lambda_{0}\right) .
$$

It is easily seen that $R M\left(B ; \lambda_{0}\right)=R M\left(E B ; \lambda_{0}\right)$, as $H_{m}\left[E B ; \lambda_{0}\right]=H_{m}\left(B ; \lambda_{0}\right]$ for each $m \in Z$. Finally, it is not difficult to prove that

$$
\hat{A}_{1}[H(M)]=\hat{A}[H(Y)], \hat{A}_{1}[M(M)]=\hat{A}[M(Y)] \text {, }
$$

(cf. (8), Lemma IV, 3.6), so $R M\left(A ; \lambda_{0}\right)=M\left(A_{1} ; \lambda_{0}\right)$. This completes the proof. 
The final theorem of this paper deals with the sublogarithmic behaviour of the reduced zero multiplicity. As follows from Theorems 2 and 4 , one has $r m_{0}\left(A B ; \lambda_{0}\right) \leqq$ $r m_{0}\left(A ; \lambda_{0}\right)+r m_{0}\left(B ; \lambda_{0}\right)$ if $H\left[A ; \lambda_{0}\right] \oplus K\left[B ; \lambda_{0}\right]=Y$, and the example at the end of (2) shows that conditions of this kind are necessary in order to obtain sublogarithmic behaviour.

Theorem 5. Let $A \in H\left(\lambda_{0}, L(Y, Z)\right)$ and $B \in H\left(\lambda_{0}, L(X, Y)\right)$ be finite meromorphic at $\lambda_{0}$, and assume that the constant terms of the Laurent expansions of $A$ and $B$ at $\lambda_{0}$ are operators with closed range and that both $r m_{0}\left(A ; \lambda_{0}\right)$ and $r m_{0}\left(B ; \lambda_{0}\right)$ are finite. If one of the following conditions

(i) $H\left[A ; \lambda_{0}\right]=\{0\}$

(ii) $K\left[B ; \lambda_{0}\right]=Y$

(iii) $H\left[A ; \lambda_{0}\right] \oplus K\left[B ; \lambda_{0}\right]=Y$

holds, then $A B$ is finite-meromorphic at $\lambda_{0}$, the constant term of its Laurent expansion at $\lambda_{0}$ has closed range, its reduced zero multiplicity is finite and

$$
r m_{0}\left(A B ; \lambda_{0}\right) \leqq r m_{0}\left(A ; \lambda_{0}\right)+r m_{0}\left(B ; \lambda_{0}\right) .
$$

Proof. All statements, except formula (19), follow from Lemma IV, 3.4 in (8). If (iii) holds, then formula (19) follows from formulae (7) and (18). Assume that (i) holds. Then $\hat{A}$ is injective. In the proof we use the following notations:

$$
\begin{aligned}
& U=\hat{A}[M(Y)], \quad V=\hat{A}[H(Y)], \quad W=\hat{A} \hat{B}[M(X)], \quad J=\hat{A} \hat{B}[H(X)], \\
& K=\hat{A}[\hat{B}[M(X)] \cap H(Y)], \quad L=\hat{A}[\hat{B}[H(X)] \cap H(Y)], \quad H=H(Z) .
\end{aligned}
$$

As $\hat{A}$ is injective we have $H \cap W \cap V=H \cap K$ and hence

$$
\begin{aligned}
& H \cap W \supset H \cap W \cap V=H \cap K \supset H \cap L \\
& H \cap W \supset H \cap J \supset H \cap L .
\end{aligned}
$$

Further

$$
\begin{aligned}
& \operatorname{dim} \frac{H \cap W}{H \cap W \cap V} \leqq \operatorname{dim} \frac{H \cap U}{H \cap V}=m_{0}\left(A ; \lambda_{0}\right), \\
& \operatorname{dim} \frac{H \cap K}{H \cap L} \leqq \operatorname{dim} \frac{K}{L}=r m_{0}\left(B ; \lambda_{0}\right), \\
& \operatorname{dim} \frac{H \cap W}{H \cap J}=r m_{0}\left(A B ; \lambda_{0}\right)
\end{aligned}
$$

and so

$$
\begin{aligned}
r m_{0}\left(A B ; \lambda_{0}\right) & =\operatorname{dim} \frac{H \cap W}{H \cap W \cap V}+\operatorname{dim} \frac{H \cap K}{H \cap L}-\operatorname{dim} \frac{H \cap J}{H \cap L} \\
& \leqq m_{o}\left(A ; \lambda_{0}\right)+r m_{0}\left(B ; \lambda_{0}\right)-\operatorname{dim} \frac{H \cap J}{H \cap L} \leqq m_{0}\left(A ; \lambda_{0}\right)+r m_{0}\left(B ; \lambda_{0}\right) .
\end{aligned}
$$

If (ii) holds then (19) may be proved by considering $B^{*} A^{*}$, as $r m_{0}\left(B^{*} ; \lambda_{0}\right)=r m_{0}\left(B ; \lambda_{0}\right)$, $r m_{0}\left(A^{*} ; \lambda_{0}\right)=r m_{0}\left(A ; \lambda_{0}\right)$ and $r m_{0}\left(B^{*} A^{*} ; \lambda_{0}\right)=r m_{0}\left(A B ; \lambda_{0}\right)$ (see (1), Proposition 5.9). 
Clearly, one may derive conditions in order that

$$
r m_{0}\left(A B ; \lambda_{0}\right)=r m_{0}\left(A ; \lambda_{0}\right)+r m_{0}\left(B ; \lambda_{0}\right) .
$$

For example, if condition (i) in Theorem 5 is satisfied, then $r m_{0}\left(A B ; \lambda_{0}\right)$ behaves logarithmically if and only if (in the notation of the proof of Theorem 5) the following conditions are satisfied

(a) $(H \cap W)+(H \cap V)=H \cap U$,

(b) $(H \cap K)+L=K$,

(c) $H \cap J=H \cap L$.

If condition (iii) in Theorem 5 is satisfied, then evidently logarithmic behaviour is obtained if and only if conditions (8) are satisfied. It is not clear whether conditions like (8), which are in terms of $H_{m}$ - and $K_{m}$-spaces rather than in terms of subspaces of $M(Z)$ or $M(Y)$, might be derived also in the case where conditions (i) and (ii) of Theorem 5 hold.

\title{
REFERENCES
}

(1) H. BART, M. A. KAASHOEK and D. C. LAY, Stability properties of finite meromorphic operator functions, Nederl. Akad. Wetensch. Proc. Ser. A 77 (1974), 217-259.

(2) H. BART, M. A. KAASHOEK and D. C. LAY, The integral formula for the reduced algebraic multiplicity of meromorphic operator-functions, Proc. Edinburgh Math. Soc. 21 (1978), 65-72.

(3) A. M. GLEASON, Finitely generated ideals in Banach algebras, J. Math. and Mech. 13 (1964), 125-132.

(4) I. C. GOHBERG and E. I. SigAL, Operator generalizations of the theorem on the logarithmic difference and of the theorem of Rouché, Mat. Sb. 84 (1971), 607-630[Russian] = Math. USSR-Sb. 13 (1971), 603-625.

(5) I. C. Gohberg and E. I. Sigal, On the root multiplicity of the product of meromorphic operator functions, Mat. Issled. 6 (1971), 34-50 [Russian]. 1966).

(6) T. Kato, Perturbation theory for linear operators (Springer, Berlin, Heidelberg, New York,

(7) M. V. KELDYSH, On the eigenvalues and eigenvectors of some classes of nonselfadjoint equation, Dokl. Akad. Nauk SSSR (1951), 11-14 [Russian].

(8) G. PH. A. THIJSSE, Decomposition theorems for finite-meromorphic operator-functions (Thesis Vrije Universiteit, Amsterdam, 1978).

(9) J. VANDEWALLE and P. DEWILDE, A local I/O structure theory for multivariable systems and its application on minimal cascade realization, preprint 1977.

(10) D. C. YoulA and P. TISSI, An explicit formula for the degree of a rational matrix (Polytech. Inst. of Brooklyn, Report PI BRI-1273-65, June 1965).

\author{
ABT. MATHEMATIK-LeHRSTUHL I \\ UNIVERSITÄT DORTMUND \\ POSTFACH 500500 \\ 4600 DORTMUND 50 \\ FED. REP. OF GERMANY
}

